\title{
Human West Nile Virus Disease Outbreak in Pakistan, 2015-2016
}

\author{
Erum Khan ${ }^{1}$, Kelli L. Barr ${ }^{2 *}$, Joveria Qais Farooqi ${ }^{1}$, Dhani Prakoso ${ }^{2}$, Alizeh Abbas ${ }^{1}$, \\ Zain Yar Khan ${ }^{1}$, Shanze Ashi ${ }^{1}$, Kehkashan Imtiaz ${ }^{1}$, Z. Aziz ${ }^{1}$, Faisal Malik' \\ John A. Lednicky ${ }^{3}$ and Maureen T. Long ${ }^{2}$
}

\begin{abstract}
'Department of Pathology and Laboratory Medicine, Aga Khan University, Karachi, Pakistan, ${ }^{2}$ Department of Comparative Diagnostic and Population Medicine, College of Veterinary Medicine, University of Florida, Gainesville, FL, United States, ${ }^{3}$ Department of Environmental and Global Health, Emerging Pathogens Institute, University of Florida, Gainesville, FL, United States
\end{abstract}

\section{OPEN ACCESS}

Edited by: Nicola Petrosillo,

Istituto Nazionale per le Malattie Infettive Lazzaro Spallanzani (IRCCS),

Reviewed by: Evandro R. Winkelmann, Columbia University, United States Lorenzo Zammarchi, University of Florence, Italy

*Correspondence: Kelli L. Barr kellilbarr@ufl.edu

Specialty section:

This article was submitted to Infectious Diseases - Surveillance,

Prevention and Treatment, a section of the journal Frontiers in Public Health

Received: 20 November 2017 Accepted: 22 January 2018 Published: 27 February 2018

Citation:

Khan E, Barr KL, Farooqi JQ, Prakoso D, Abbas A, Khan ZY, Ashi S, Imtiaz K, Aziz Z, Malik F, Lednicky JA and Long MT (2018) Human West Nile Virus Disease Outbreak in Pakistan, 2015-2016.

Front. Public Health 6:20. doi: 10.3389/fpubh.2018.00020
Like most of the world, Pakistan has seen an increase in mosquito-transmitted diseases in recent years. The magnitude and distribution of these diseases are poorly understood as Pakistan does not have a nation-wide system for reporting disease. A cross-sectional study to determine which flaviviruses were causing of arboviral disease in Pakistan was instituted. West Nile virus (WNV) is a cause of seasonal fever with neurotropic findings in countries that share borders with Pakistan. Here, we describe the active and persistent circulation of WNV in humans in the southern region of Pakistan. This is the first report of WNV causing neurological disease in human patients in this country. Of 997 enrolled patients presenting with clinical features suggestive of arboviral disease, 105 were positive for WNV IgM antibodies, and 71 of these patients possessed WNV-specific neutralizing antibodies. Cross-reactivity of WNV IgM antibodies with Japanese encephalitis virus (JEV) occurred in 75 of these 105 patients. WNV co-infections with Dengue viruses were not a contributing factor for the severity of disease. Nor did prior exposure to dengue virus contribute to incidence of neurological involvement in WNV-infected patients. Patients with WNV infections were more likely to present with altered mental status, seizures, and reduced Glasgow Coma scores when compared with JEV-infected patients. Human WNV cases and vector numbers exhibited a temporal correlation with climate.

Keywords: West Nile virus, Dengue virus, Japanese encephalitis virus, encephalitis, arboviral disease

\section{INTRODUCTION}

West Nile virus (WNV) is a single-stranded, enveloped positive-sense RNA flavivirus that is maintained in nature by an enzootic cycle between birds and blood-feeding Culex mosquitoes (family Culicidae) (1). Culex spp. are widely distributed over the world and have been reported to feed on birds, humans, horses, and other mammals (2). Outbreaks of WNV disease have been reported in Europe, America, Africa, and parts of Asia (3-5). Serological evidence in humans and vector competence for WNV in Pakistan has been reported as early as $1982(6,7)$. In recent years, WNV has been identified in horses and humans in Pakistan and its neighboring countries, Iran, Afghanistan, and India as a cause of human encephalitic disease (8-13). Surveillance studies in these surrounding 
countries report WNV circulation in several Culex species as well as several wild, migratory, and domestic birds and water fowl (14-19).

With the dissolution of the Pakistan Federal Ministry of Health in 2011, reporting and surveillance for arboviral diseases have become largely non-existent and, when performed, is highly localized and mostly funded by foreign granting agencies. Pakistan is at a continual risk of suffering large epidemics of vector-borne diseases for a variety of reasons. Geopolitical instability, annual flooding, and poor health care infrastructure lead to frequent internal human and animal displacement. Moreover, the construction of super highways and shipping ports for efficient travel between Africa and China has left Pakistan vulnerable to the introduction of foreign diseases. Pakistan has recently witnessed an increased burden of arboviral infections, primarily from Crimean-Congo hemorrhagic fever virus, WNV, Chikungunya virus (CHIKV), and Dengue viruses (DENVs) affecting the economic, social, and political aspects of people's lives (7, 20-22). More recently, active circulation of DENV, WNV, and $\mathrm{CHIKV}$ has been reported in humans living in the Punjab province of Pakistan (22).

Pakistan lacks a holistic surveillance system for arbovirus infections; therefore, there are limited data regarding types of vectors circulating, their temporal trends, and their correlations with climatic factors and human/animal cases. In light of the heightened alert of epidemic outbreaks and the global spread of arboviruses like WNV, CHIKV, and Zika virus, active surveillance for arboviruses in Pakistan is desperately needed for effective implementation of preventive and control strategies. Here, we present results of a multiyear seroprevalence and mosquito surveillance project initiated in 2015 that analyzed human arboviral exposure, mosquito vectors, and their correlation with climatic factors.

\section{MATERIALS AND METHODS}

\section{Patient Enrollment}

A cross-sectional, observational study was performed to identify which arboviruses [DENV, WNV, and Japanese encephalitis virus (JEV)] were the cause of acute undifferentiated febrile illness in selected basic health units and/or district hospitals of the Sindh region of Pakistan (8). Patients were enrolled under informed consent procedures, which were reviewed and approved by the Ethics Review Committee at Aga Khan University (3183-PATERC-14) and the Institutional Review Board at the University of Florida (201500908). All enrolled subjects gave written informed consent in accordance with the Declaration of Helsinki. Patients presenting with the CDC clinical description of arboviral disease including findings of rash, headache, arthralgia, myalgia, gastrointestinal distress, acute hemorrhagic fever, acute flaccid paralysis, encephalitis, meningitis, and/or unexplained fever were recruited (23). Patients younger than 10 years and older than 90 years were excluded. Briefly, all patients were tested for DENV NS1 antigen unless affected primarily by neurologic abnormalities. All NS1-negative sera were evaluated for exposure to WNV or JEV via IgM capture ELISA for JEV and WNV.

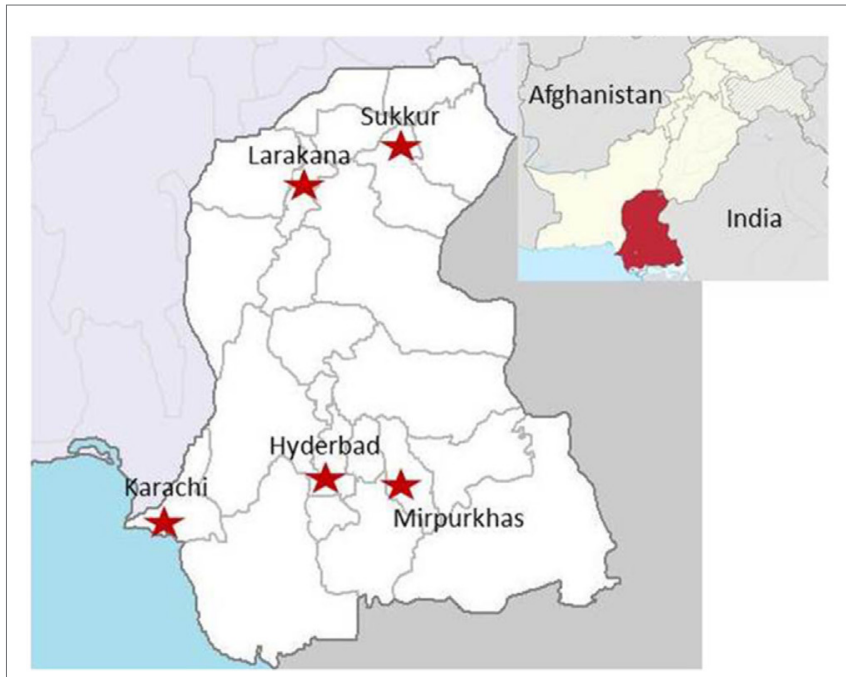

FIGURE 1 | Locations of the patient enrollment sites in the Sindh region of Pakistan (8). Sites included medical colleges, teaching hospitals, and civil hospitals.

Five study sites were established, and personnel were trained throughout the Sindh province in Pakistan (Figure 1) (8). These sites included medical colleges, teaching hospitals, and civil hospitals.

\section{Diagnostic Criteria}

Patients were confirmed positive for virus exposure according to CDC criteria, which requires "virus-specific IgM antibodies in serum with confirmatory virus-specific neutralizing antibodies in the same or a later specimen" (23). Patients were classified as a suspect case if they had virus-specific IgM antibodies in serum but with a neutralizing titer of less than $50 \%$ at the highest concentration of serum. For cross-reactive specimens, patients were confirmed positive when they had virus-specific IgM antibodies and neutralizing antibodies in the same specimen that exhibited the highest level of neutralization for one of the viruses in question. Cross-reactive patients were classified as suspect according to the greatest amount of virus-specific IgM antibodies. Patients were considered positive for DENV co-infection if they had DENV-specific NS1 antigen. Patients were considered as having prior DENV exposure if they exhibited greater than $80 \%$ neutralization at the 1:2560 serum dilution.

\section{NS1 and IgM ELISA Testing for Flaviviruses}

Primary DENV screening in patients was performed using a commercial ELISA (Panbio Dengue Early Rapid Test NS1 antigen capture test, Alere, Waltham, MA, USA) following manufacturer's instructions. For WNV and JEV, a commercial IgM capture ELISA for work with diagnostic specimens in BSL-2 facilities (InBios WNV Detect ${ }^{\mathrm{TM}}$ and JEV Detect ${ }^{\mathrm{TM}}$, InBios, Seattle, WA, USA) was used following the manufacturer's instructions. 


\section{RT-PCR}

West Nile virus and JEV primers and probes were used to detect the NS2 gene (24). The samples of all WNV and/or JEV IgMpositive samples and mosquito pools were screened. Briefly, RNA was isolated from mosquito pools, human CSF (where received, patients with neurological symptoms only), and serum samples using a commercial virus RNA extraction kit (QIAamp viral RNA kit, Qiagen, Valencia, CA, USA). Mosquito pools were homogenized in PBS to form a 1:10 (v/v) homogenate using sterile pestles in $1.5-\mathrm{ml}$ polypropylene tubes. All samples were stored at $-80^{\circ} \mathrm{C}$ until use.

RNA was reverse transcribed to cDNA (iScript cDNA Synthesis Kit, Bio-Rad, Hercules, CA, USA), and PCR was performed. For the WNV PCR, 1,000 nM of forward and reverse primers and $200 \mathrm{nM}$ of fluorogenic probe were used, and for the JEV PCR, $375 \mathrm{nM}$ of forward and reverse primers and $250 \mathrm{nM}$ of fluorogenic probe were used (Biosearch Technologies, Petaluma, CA, USA) in a $20-\mu$ total reaction volume. Standard cycling conditions consisted of once at $95^{\circ} \mathrm{C}$ for 2 min followed by 40 cycles of $95^{\circ} \mathrm{C}$ for $15 \mathrm{~s}$ and $60^{\circ} \mathrm{C}$ for $1 \mathrm{~min}$.

\section{Plaque Reduction Neutralization Testing (PRNT)}

A PRNT was performed to determine the neutralizing capacity of patient serum. Viruses used included the following: WNV (NY99), DENV-1 (TS-SMAN), DENV-2 (NGC), DENV-3 (H87), DENV-4 (H241), and JEV (SA-14-8). All PRNT assays were performed side by side under BSL-3 containment to ensure continuity of data. Four-fold dilutions of heat-inactivated patient serum were mixed with an equal volume of MEM (Corning, Tewksbury, MA, USA) containing 50-100 plaque-forming units of virus and incubated for $1 \mathrm{~h}$ at $37^{\circ} \mathrm{C}$. After incubation, this mixture was inoculated onto confluent Vero E6 cells in 12 -well plates, which were then incubated for $1 \mathrm{~h}$ at $37^{\circ} \mathrm{C}$. After incubation, the inoculum was removed; and an overlay of MEM with $0.5 \% \mathrm{wt} / \mathrm{vol}$ methylcellulose, $2.5 \% \mathrm{FBS}$, non-essential amino acids, penicillin, streptomycin, and amphotericin B was added to the wells; and the plates were incubated until plaques were visible (3-10 days). For visualization of plaques, the methylcellulose overlay was removed, and cells were stained with a fresh mixture of $50 \%$ methanol, $43 \%$ ethanol, $7 \%$ acetic acid, and $0.1 \%$ wt/vol Comassie blue and plaques were counted. The antibody titer was determined as the highest serum dilution that neutralized $50 \%$ of the virus inoculum.

\section{Vector Surveillance}

Eight sites (1-8) were selected in Karachi for mosquito trap placement (Figure 2) based on their close vicinity to trees/shrubbery, water, and human activity. The area was spread over $0.34 \mathrm{~km}^{2}$ and landscaped with high-rise buildings, lakes, water pools, and intermittent green patches. The average distance between the sites was $200 \mathrm{~m}$.

Three types of mosquito traps were used including the CDC Miniature Light Trap (Model 512), the BG-Sentinel ${ }^{\mathrm{TM}}$ (Biogents GmbH, Regensburg, Germany) and the CDC Gravid trap (John W. Hock Company, Gainesville, Florida, model 171). The traps were set per manufacturers' recommendations. The CDC light

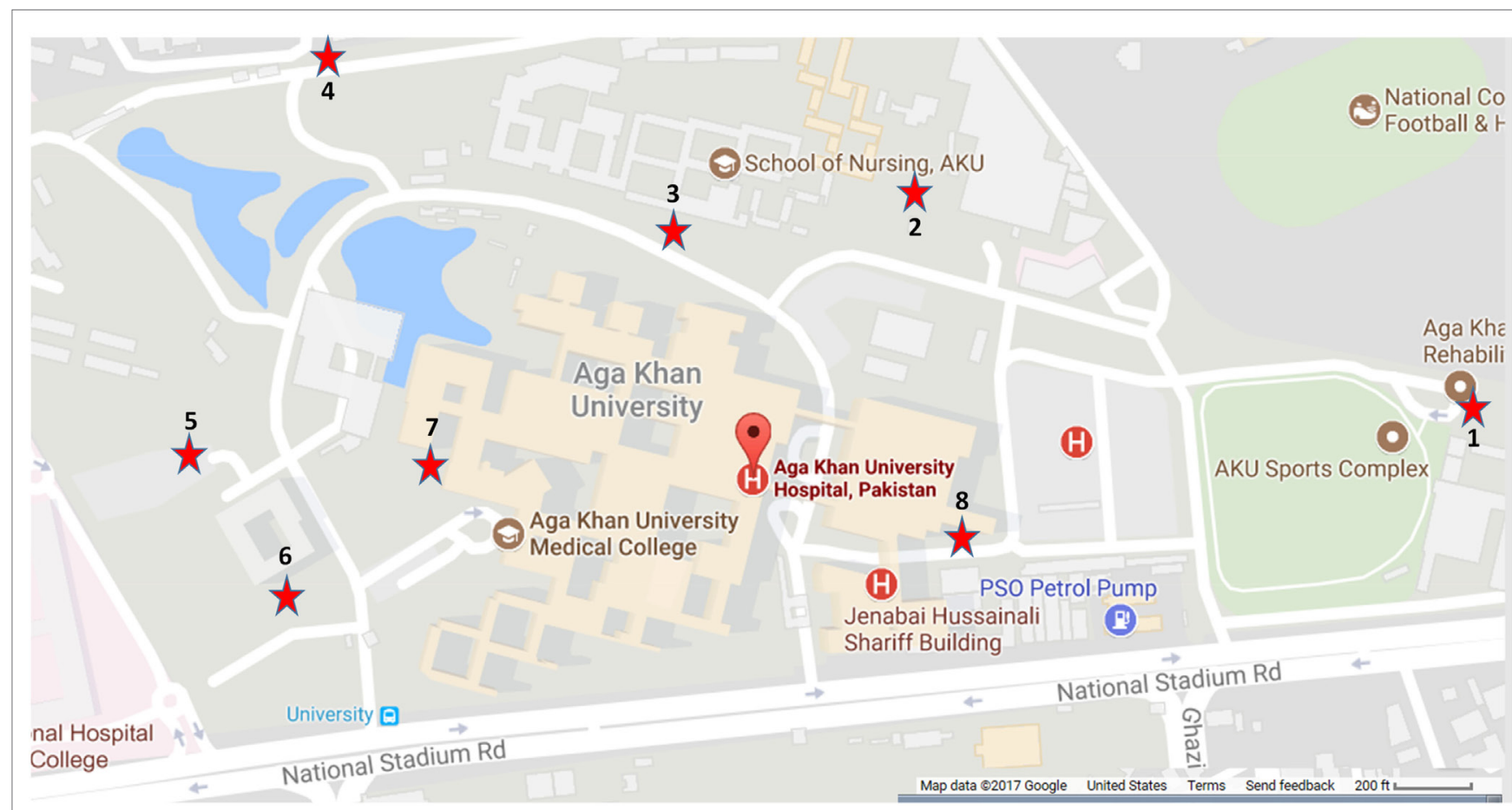

FIGURE 2 | Locations of the mosquito traps used for vector collection, May-November 2015. Sites were selected based on their close vicinity to trees/shrubbery, water, and human activity. The area encompassed $0.34 \mathrm{~km}^{2}$ and included high-rise buildings, lakes, water pools, and green patches. 
trap was hung approximately $2 \mathrm{~m}$ above the ground. The BG sentinel trap was baited with the BG-Lure attractant supplied by the manufacturer and placed near an area sheltered from wind and direct sunlight. The Gravid Trap infusion was prepared by adding $3 \mathrm{l}$ of water to dry grass hay. These traps were connected to a $12 \mathrm{~V}$ rechargeable battery.

Mosquito surveillance activity began in May 2015 and ended in November 2015. Traps were placed near identified locations for four consecutive nights (Monday to Thursday) from 4:00 p.m. to 9:00 a.m. Each set of traps was placed at one site for 1 week and then rotated to another site. The mosquitoes were collected daily and taken directly to the research laboratory, and mosquitoes were placed on a chilled Petri dish and separated and differentiated according to sex and species. Female mosquitoes species were further separated according to blood and non-blood fed. All identified mosquitoes were quantified and aliquoted and stored at $-80^{\circ}$. For each RT-PCR reaction, RNA was extracted from individual pools of 30 mosquitoes. Mosquito grinding and RNA extraction was performed using Qiagen viral RNA extraction kit.

\section{Environment Monitoring of Temperature and Humidity Charts}

The Pakistan Meteorological Department, Karachi head-office graciously shared their daily temperature, humidity, and precipitation monitoring data. Mean temperature, humidity, and precipitation were calculated and recorded. Monthly data compilations were correlated with the number of mosquitoes collected from traps and confirmed human WNV cases.

\section{Statistical Analysis}

Statistical analyses were performed on clinical data using MedCalc version 17.9.7-64-bit. Logistic regression for dichotomous independent variables was performed. Odds ratios were calculated with $95 \%$ confidence intervals. Ratios with a $p$ less than 0.05 were considered significant. Pearson's correlation coefficients, and associated $p$ values were calculated to identify potential relationships between different variables in the data set.

\section{RESULTS}

\section{WNV Is a Cause of Febrile Illness in Patients with Undifferentiated Fever in Pakistan}

West Nile virus exposed patients were enrolled from all five sites beginning in May 2015 and continuing through December 2016 (Figure 3). Peaks in human case were observed during September and October, which is consistent with the incidence of WNV reported in other endemic areas like Europe, the United States, and the Mediterranean region (Figure 3). Clinical specimens were confirmed positive based on the CDC guidelines that require detection of virus-specific IgM antibodies with virus-specific neutralizing antibodies in the same or later specimen (23). Of a total of 997 patients enrolled, based on the CDC criteria for clinical manifestations of arboviral disease, 105 patients tested positive for WNV IgM. Twenty-eight (26.6\%) of these patients were positive for WNV IgM only (Table S1 in Supplementary Material). Seventy-nine (75.23\%) patients exhibited positive IgM for both WNV and JEV (Table S1 in Supplementary Material). Eight (7.5\%) of these patients were also positive for DENV NS1 antigen (Table S1 in Supplementary Material).

The 105 samples that were positive for WNV IgM were evaluated for neutralizing antibodies specific for JEV and WNV via PRNT. Thirty-six (33.96\%) exhibited greater neutralization for

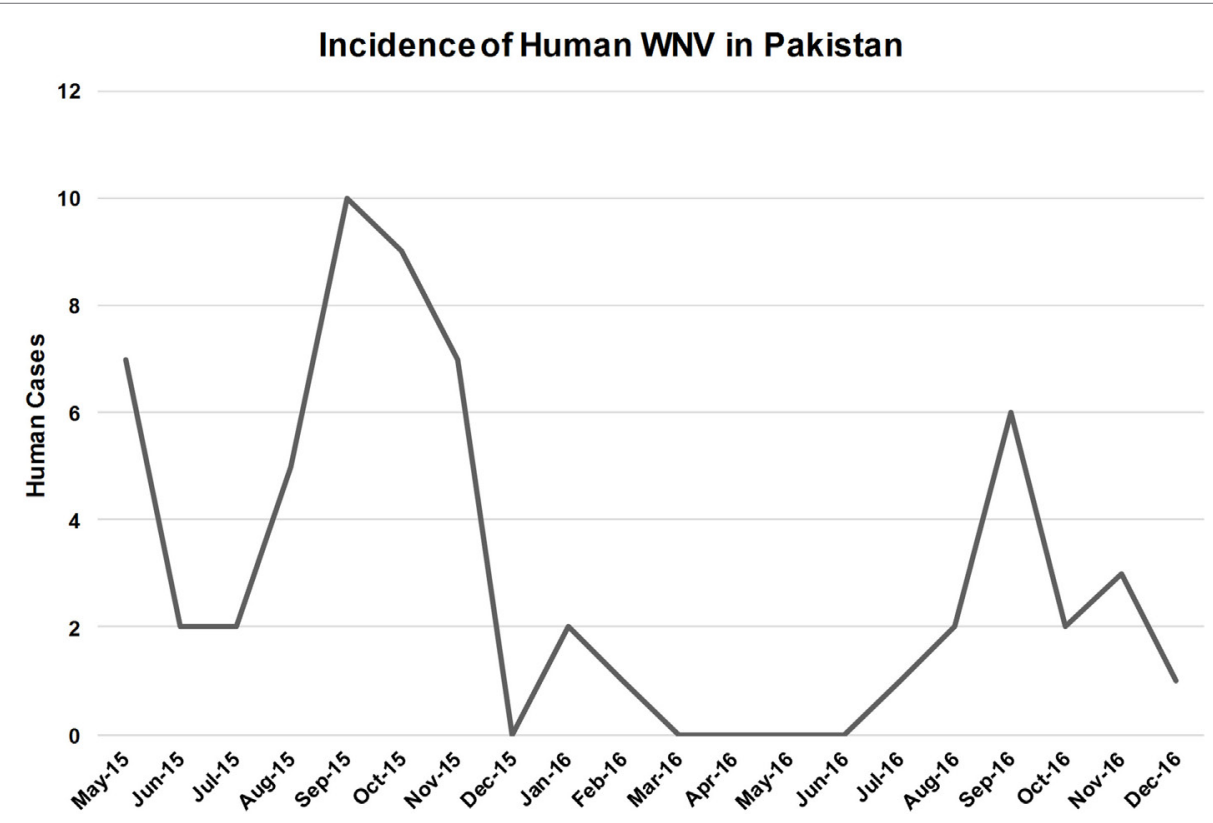

FIGURE 3 | Number of patients with both West Nile virus (WNV)-specific IgM and neutralizing antibodies. Data include patient specimens from all five study sites over a 2-year period. 
WNV than JEV when the minimum neutralization was set at 80\% (Table S1 in Supplementary Material). Fifty-nine (55.66\%) patients exhibited greater neutralization of WNV than JEV when the minimum neutralization was decreased to 50\% (Table S1 in Supplementary Material). In the absence of neutralizing antibodies, patients were classified as probable exposures for the virus for which they possessed the highest IgM activity. Seventeen (16.1\%) individuals had greater neutralizing activity for JEV than WNV (Table S1 in Supplementary Material). Twenty-nine (27.36\%) patients did not exhibit significant neutralization of either WNV or JEV (Table S1 in Supplementary Material). CSF samples from two patients who presented with signs and symptoms suggestive of viral encephalitis/meningitis were tested by RT-PCR for WNV; however, none of the samples were positive for WNV (data not shown).

\section{Clinical Features of Human WNV Infection}

Of the $105 \mathrm{WNV}$ IgM-positive patients, $61.9 \%$ were male $(n=65$; Table 1). The mean age was 31 years; however, patients from 10 to 86 were enrolled (data not shown). Clinical findings at time of presentation included headache, body ache, and nausea/vomiting and were seen in most patients enrolled. The average duration of fever at time of presentation was $4( \pm 2)$ days. Thirty-nine $(n=41)$ presented with signs and symptoms suggestive of central nervous system (CNS) involvement that included altered consciousness, vertigo, seizures, limb weakness, decreased Glasgow Coma scores, and encephalitis (Table 1). For the sake of simplifying data analysis, acute disseminated encephalomyelitis, meningitis, and meningoencephalitis were all classified as encephalitis.

Although males comprised $73.5 \%$ of the WNV IgM positive and $67 \%$ of the WNV confirmed population, this variable was not significant (Table 1). Clinical symptoms suggestive of WNV neuroinvasive disease were not always indicative of WNV exposure. WNV confirmed individuals were 4.25 times more likely to present with altered mental status $(p=0.0058)$ and twice as likely to have seizures, hemorrhage, and encephalitis (Table 1). Other symptoms of WNV neuroinvasive disease such as stiff neck, vertigo, limb weakness, and reduced Glasgow Coma Scale were not significant predictors of exposure and were just as likely to be observed in individuals negative for WNV exposure (Table 1). Over half of WNV-exposed individuals were younger than 30 years, and $82 \%$ were younger than 40 years (Figure 4). Although most patients with encephalitis were in the 20-30 age group, the percentage of encephalitis was highest in the very young or in the 50-60 and 60-70 age groups (Figure 4).

When comparing the symptoms of WNV- and JEV-exposed individuals, several features were unique for each virus. Patients with JEV exposure were 7.16 times more likely to present with GI distress including nausea and vomiting $(p=0.0053)$ and 5.5 times more likely to be male ( $p=0.0445$; Table 2 ). Persons with JEV exposure were also nearly five times more likely to exhibit thrombocytopenia than WNV-exposed patients $(p=0.0438$; Table 2). However, altered mental status was not reported for patients with JEV exposure and was 4.25 times more likely to be identified in patients with WNV exposure ( $p=0.043$; Table 2).

Unfortunately, immune status ratios (ISRs) for the WNV and JEV IgM kits did not correlate with neutralization via PRNT ( $p=0.76 \mathrm{WNV}, p=0.12$ JEV; Table S1 in Supplementary Material). Many WNV and JEV ISR values indicated positive exposure, but the samples had little to no neutralizing activity (Table S1 in Supplementary Material). Moreover, several WNV ISR values indicated WNV exposure, but when assayed via PRNT, these patients had greater neutralizing activity for JEV than WNV (Table S1 in Supplementary Material). In the absence of neutralizing antibodies, specimens were classified as suspect/

TABLE 1 | Incidence of symptoms of suspect and confirmed West Nile virus (WNV)-positive patients compared to WNV-negative patients presenting with clinical features suggestive of arboviral disease.

\begin{tabular}{|c|c|c|c|c|}
\hline Symptom & WNV confirmed $(n)$ & WNV IgM+ (n) & WNV confirmed, OR $(95 \% \mathrm{Cl})$ & WNV suspect, OR $(95 \% \mathrm{Cl})$ \\
\hline Male & $67 \%(40)$ & $73.5 \%(64)$ & $0.54(0.22-1.31)$ & $0.24(0.05-1.13)$ \\
\hline Eye pain & $8.4 \%(5)$ & $9 \%(8)$ & $1.33(0.30-5.87)$ & $1.59(0.18-13.79)$ \\
\hline Fever $\geq 38^{\circ} \mathrm{C}$ & $44 \%(26)$ & $43.6 \%(38)$ & $1.33(0.61-2.90)$ & $1.52(0.55-4.25)$ \\
\hline Hemorrhage & $18.6 \%(11)$ & $19.5 \%(17)$ & $2.09(0.68-6.44)$ & $4.11(0.51-33.12)$ \\
\hline Rash & $11.8 \%(7)$ & $23.7 \%(14)$ & $1.24(0.44-3.52)$ & $0.74(0.21-2.56)$ \\
\hline Nausea & $47.4 \%(28)$ & $59.7 \%(52)$ & $0.40(0.18-0.89)$ & $0.29(0.09-0.95)$ \\
\hline Headache & $42.3 \%(25)$ & $43.5 \%(38)$ & $1.04(0.48-2.28)$ & $1.29(0.46-3.61)$ \\
\hline Myalgia & $35 \%(21)$ & $42.5 \%(37)$ & $0.66(0.30-1.45)$ & $0.89(0.33-2.46)$ \\
\hline Arthralgia & $15 \%(9)$ & $13.8 \%(12)$ & $1.48(0.46-4.75)$ & $0.78(0.19-3.13)$ \\
\hline Hypertension & $10.7 \%(6)$ & $16 \%(14)$ & 0.89 (0.29-2.68) & $0.54(0.15-1.93)$ \\
\hline Altered mental status & $28.3 \%(17)$ & $24 \%(21)$ & $4.25(1.32-13.69)$ & $5.45(0.68-43.44)$ \\
\hline Seizures & $15 \%(9)$ & $13.8 \%(12)$ & $2.58(0.66-10.14)$ & $2.64(0.32-21.79)$ \\
\hline Limb weakness & $5 \%(3)$ & $6.9 \%(6)$ & $0.56(0.12-2.65)$ & 0.52 (0.09-2.93) \\
\hline Encephalitis & $21.7 \%(13)$ & $22.9 \%(20)$ & $1.62(0.62-4.24)$ & $2.75(0.59-12.88)$ \\
\hline Thrombocytopenia & $61.7 \%(37)$ & $71.2 \%(62)$ & $0.55(0.23-1.31)$ & 0.36 (0.09-1.34) \\
\hline Lymphocytosis & $33.3 \%(20)$ & $36.8 \%(32)$ & $1.17(0.52-2.65)$ & $0.68(0.24-1.87)$ \\
\hline Reduced Glasgow Coma Scale & $10.7 \%(6)$ & $10.7 \%(9)$ & $1.96(0.48-8.06)$ & $423 E+006$ \\
\hline Any neurological symptom & $40.7 \%(24)$ & $47.1 \%(41)$ & $1.65(0.75-3.62)$ & $3.58(1.10-11.66)$ \\
\hline Elevated AST & $92.6 \%(25)$ & $61 \%(63)$ & $0.95(0.80-1.12)$ & $2.00(0.49-8.24)$ \\
\hline Elevated ALT & $68.4 \%(26)$ & $62.5 \%(42)$ & $1.2(0.47-3.34)$ & $1.05(0.83-1.13)$ \\
\hline
\end{tabular}

Patients with both WNV-specific IgM and neutralizing antibodies were considered confirmed, and patients with only lgM antibodies were considered suspect. Bold values indicate statistical significance for the odds ratio $(p<0.05)$. 
probable exposure based on whichever virus exhibited the greatest ISR values.

\section{WNV and JEV IgM Responses Are Highly Cross-Reactive}

A subset of 28 patients with JEV and WNV IgM antibodies were evaluated for neutralization of DENV. These patients were all positive for WNV, DENV, and JEV IgM. PRNTs were performed for these patients against DENV 1-4, WNV, and JEV (Table S1 in Supplementary Material). All but three patients had at least $80 \%$ neutralizing activity against at least one DENV serotype at antibody titers of 1:40 (Table S1 in Supplementary Material). Two of the three non-DENV neutralizing patients did not exhibit neutralizing activity against WNV or JEV despite having encephalitis

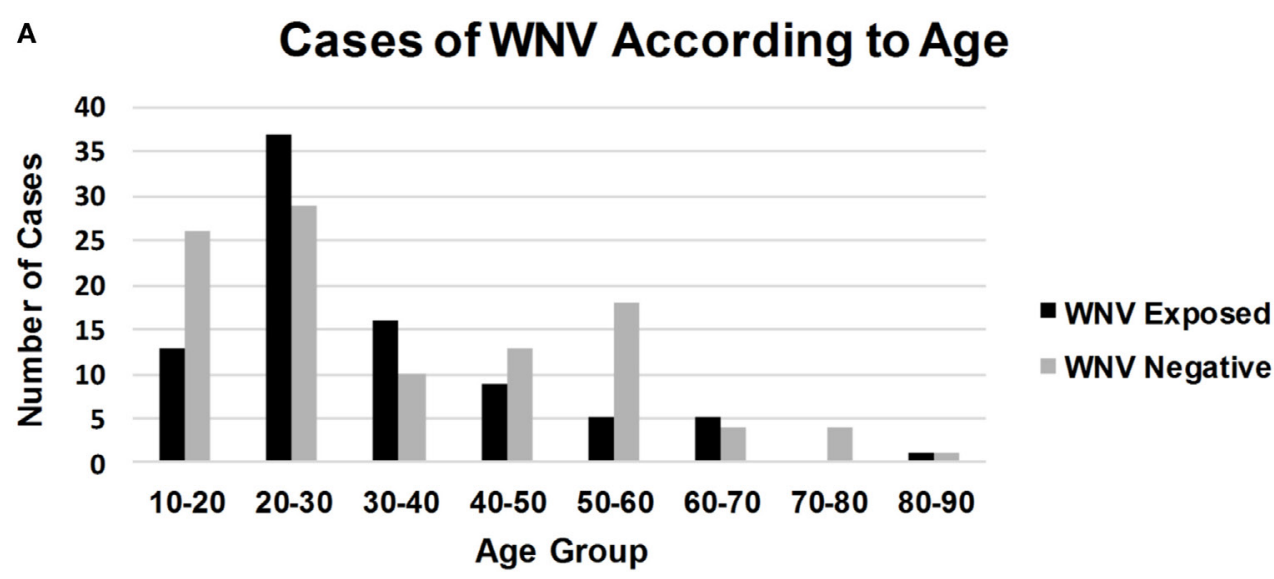

Cases of Encephalitis According to Age

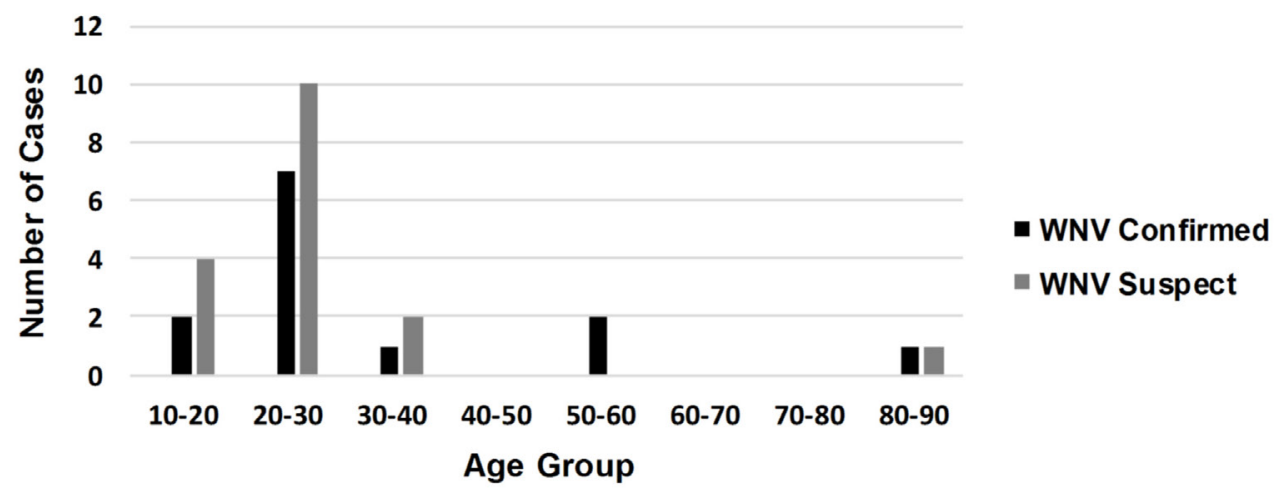

B

Percentage of Cases with Neurological Symptoms

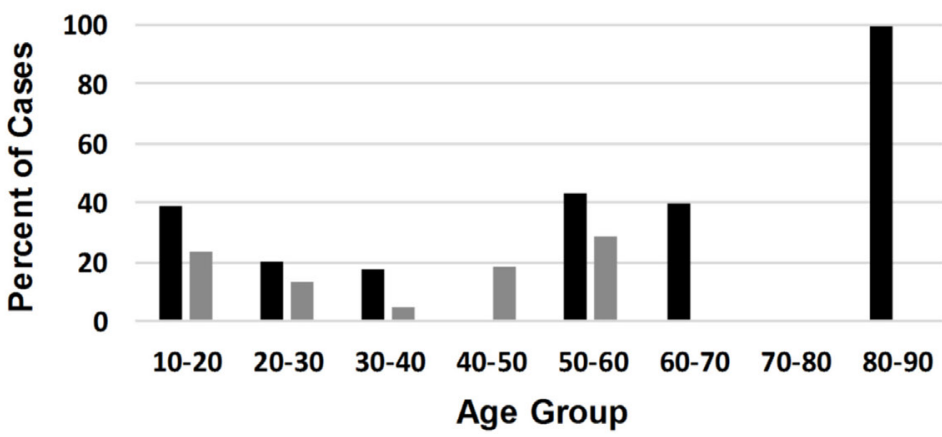

- WNV Confirmed

WNV Suspect

FIGURE $4 \mid$ Continued 
c Incidence of Encephalitis According to Age

12

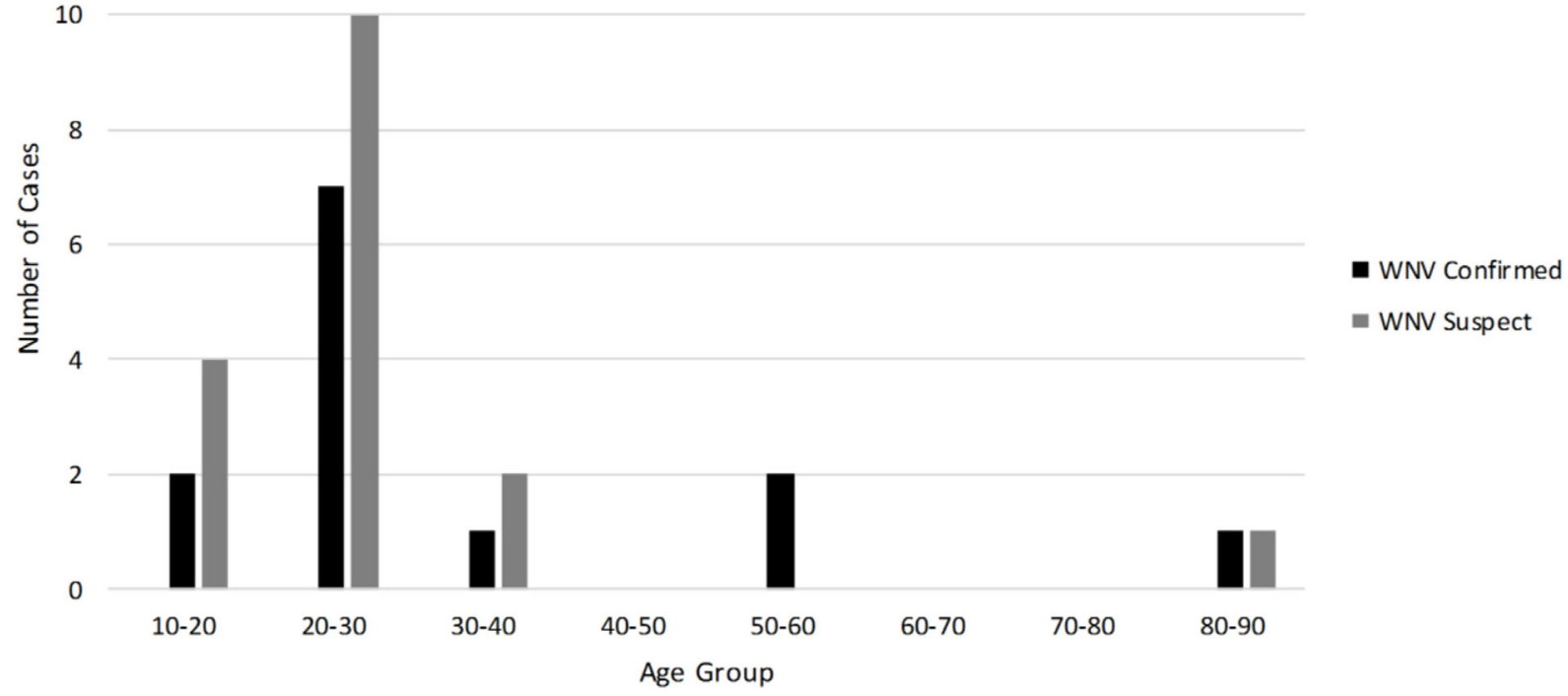

FIGURE 4 | A graphical representation of how WNV infections (A), WNV encephalitis (B), and percentage of cases with neurological symptoms (C) occurred in different age groups.

TABLE 2 | Incidence of symptoms in West Nile virus (WNV) and Japanese encephalitis virus (JEV) confirmed cases in patients presenting with clinical features suggestive of arboviral disease.

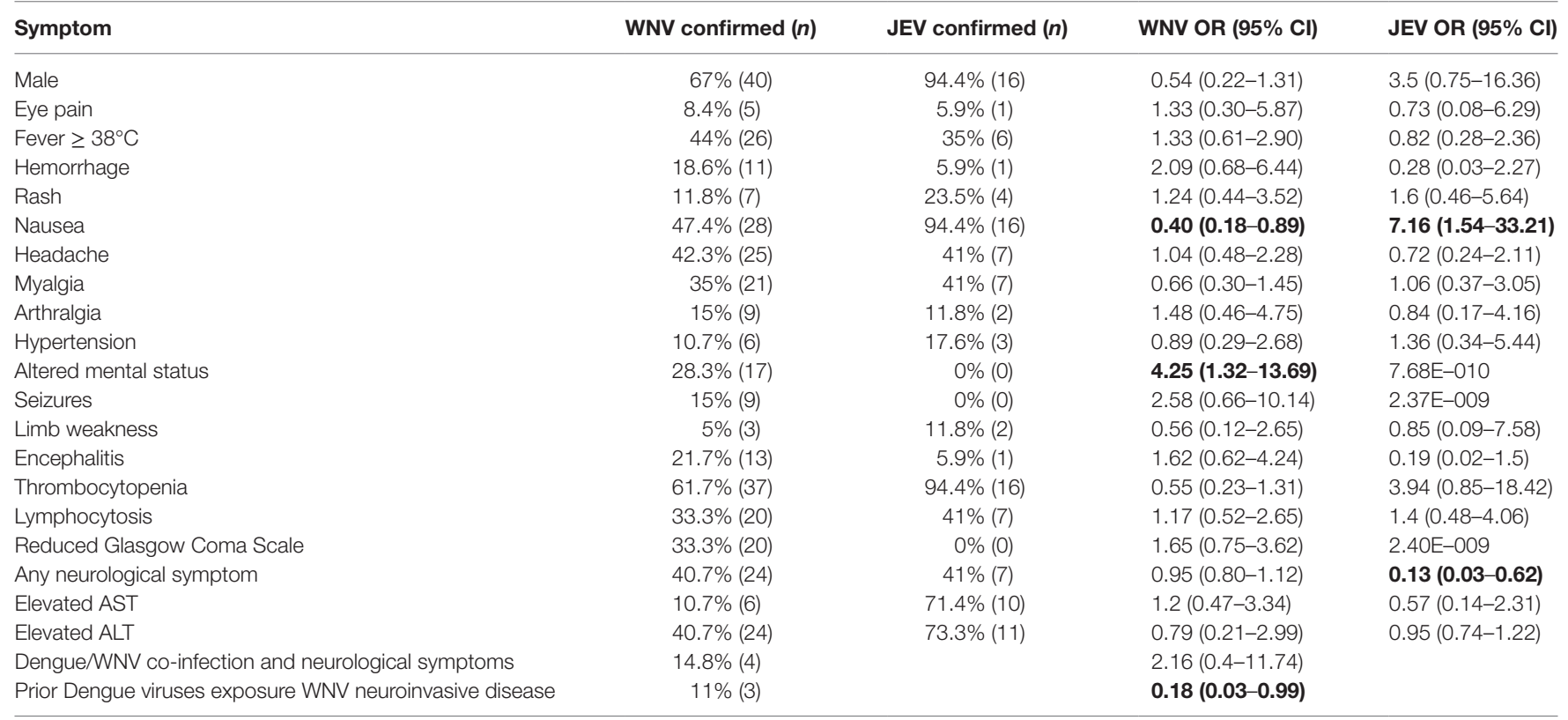

Patients with both virus-specific IgM and neutralizing antibodies were considered confirmed. For cross-reactive patients, exposure was confirmed for the virus with the greatest Plaque Reduction Neutralization Testing titer. Bold values indicate statistical significance $(p<0.05)$.

and limb weakness and positive IgM antibodies (Table S1 in Supplementary Material). The remaining patient had neutralizing antibodies to WNV only and presented with altered mental status and meningitis. Sixteen of the 28 patients exhibited serological profiles indicative of prior DENV infections with DENV neutralization of at least $80 \%$ at the 1:2560 serum dilution (Table S1 in Supplementary Material). Three of these patients exhibited symptoms of neuroinvasive disease, but having prior exposure to DENV was not a contributing factor $(p=0.0238$; Table S1 in Supplementary Material). Here again, DENV ISR values did 


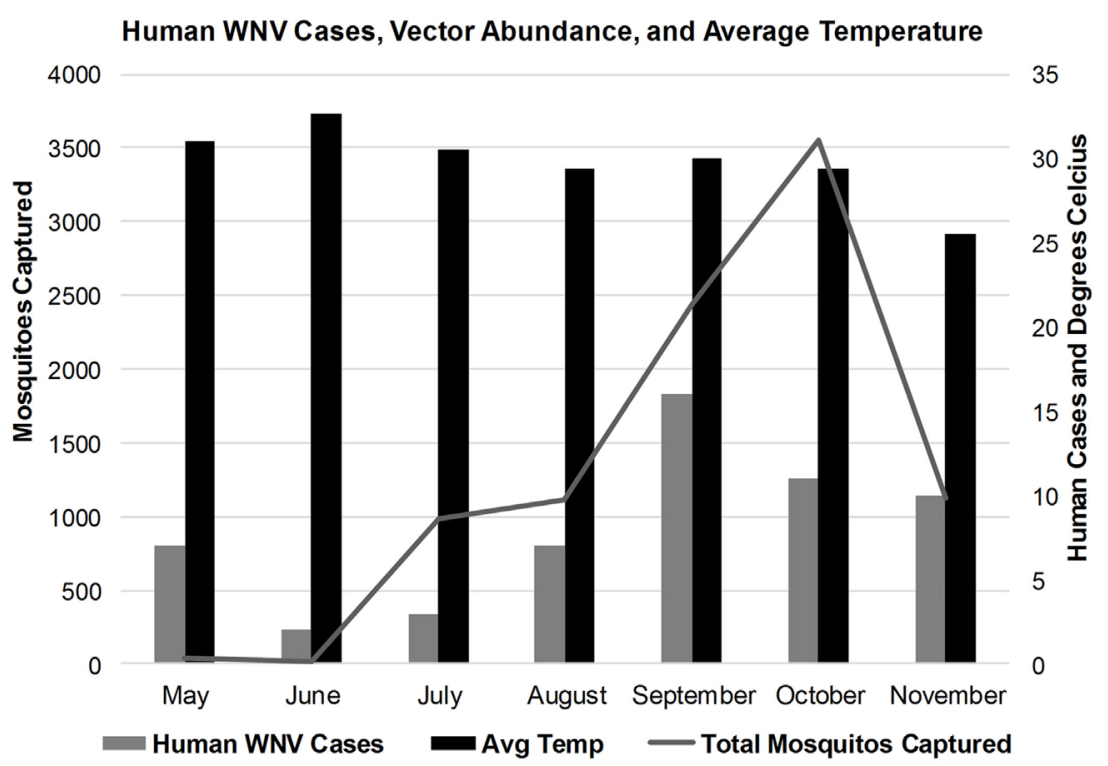

FIGURE 5 | Correlation of human West Nile virus cases, vector abundance, and average monthly temperatures in Karachi, Pakistan.

not correlate with neutralizing activity $(p=0.2931$ DENV1, $p=0.3157$ DENV2, $p=0.4099$ DENV3, $p=0.6602$ DENV4) nor were the ISR values dependent on whether the patient had a primary or secondary DENV exposure $(p=0.9861$; Table S1 in Supplementary Material).

\section{DENV/WNV Co-infections Are Associated with Neurological Symptoms}

Dengue virus NS1 antigen was detected in eight patients with WNV-specific IgM and neutralizing antibodies. Four patients exhibited symptoms of neuroinvasive disease and were twice as likely to exhibit neurological symptoms than patients without DENV co-infection (Table S1 in Supplementary Material).

\section{Vector Abundance Correlates with Temperature and Precipitation}

Temperature recordings for Karachi during 2015 showed an increase in temperatures in May through October with average temperatures between 31 and $30^{\circ} \mathrm{C}$. Peak temperatures occurred in June (average $=33 \mathrm{C}^{\circ}$; Figure 5). During this period, Karachi experienced the worst heat wave on record for June with temperatures reaching $43^{\circ} \mathrm{C}$. During this time, significantly fewer mosquitoes were collected (Figure 5). The average humidity showed an upward trend starting in May that continued through October. During November, Karachi experienced a fall in average temperatures by $3-4^{\circ}$ and a drop in relative humidity by $20 \%$ compared to the preceding months (Figure 5).

The average number of mosquitoes collected in the traps correlated with peak temperatures of the city when the heat waves
TABLE 3 | Numbers, species, and sex of mosquitoes caught in three types of trap.

\begin{tabular}{llccc}
\hline Trap & Genus & Male & Female & Total \\
\hline CDC gravid & Culex & 2,466 & 3,365 & 5,831 \\
BG sentinel & Culex & 1,566 & 1,315 & 2,881 \\
CDC light & Culex & 62 & 91 & 153 \\
CDC gravid & Anopheles & 9 & 18 & 27 \\
BG sentinel & Anopheles & 14 & 13 & 27 \\
CDC light & Anopheles & 0 & 0 & 0 \\
CDC gravid & Aedes aegypti/Aedes albopictus & 1 & 6 & 7 \\
BG sentinel & A. aegypti/A. albopictus & 2 & 3 & 5 \\
CDC light & A. aegypti/A. albopictus & 0 & 0 & 0 \\
\hline
\end{tabular}

were subtracted from the analysis ( $r=0.8067)$ (Figure 4). A total of 8,931 mosquitoes were collected during the study period. The majority of mosquitoes belonged to the Culex species (Table 3). $47.5 \%$ of the total number of mosquitoes were male Culex, while female Culex represented $51.8 \%$ of the total (Table 3). Anopheles (male $0.27 \%$; female $0.34 \%$ ) and Aedes (male $0.03 \%$; female $0.097 \%$ ) species accounted for very small percentages of the total (Table 3). Mosquito numbers were lowest in the months of May and June $(n=52)$; however, numbers increased significantly during July $(n=984)$ and August $(n=1,110)$ with a peak in September $(n=2,434)$ and October $(n=3,553$; Figure 5). A sharp decline in mosquito numbers was recorded in November ( $n=1,125$; Figure 5). It was during the month of September that $\mathrm{WNV}$ was detected in a pool of 30 Culex mosquitoes that were trapped at location 8 (Figure 2), which is near the entrance to the offices for the Aga Khan University Hospital.

Of the three types of traps used in this study, the CDC Gravid Trap was the most successful in capturing Culex spp., catching $65.7 \%$ of all Culex spp. (Table 3). This was twice as effective as the 
BG Sentinel and 38 times more effective than the CDC light Trap (Table 3). For Anopheles and Aedes species, the CDC Gravid Trap and the BG-Sentinel performed the same (Table 3). The CDC Light Trap did not capture any Anopheles or Aedes species and overall was the least successful, contributing only $2 \%$ to the total number of mosquitoes caught (Table 3 ).

\section{DISCUSSION}

Diagnosis and appropriate treatment of arboviral disease is problematic in areas where these pathogens co-circulate. Overlapping clinical presentation combined with serological cross-reactivity render definitive diagnosis expensive and cumbersome. Furthermore, secondary infections with these types of viruses can exacerbate clinical symptoms and complicate interpretation of diagnostic tests. Laboratory confirmation remains a major challenge, and RT-PCR is not very useful in diagnosis of WNV or JEV as viremia occurs 3-4 days before the onset of clinical symptoms. Given that DENV, JEV, and WNV are flaviviruses, there is serological cross-reactivity and absolute confirmation requires the most cumbersome and highly skilled plaque reduction assay. This type of infrastructure is lacking in many labs and medical facilities in Pakistan.

The data herein emphasize that viral IgM is not indicative of exposure. Patients positive for JEV IgM were usually positive for WNV IgM and vice versa. Even more troubling was that these cross-reactive samples were also invariably positive for DENV IgM (Table S1 in Supplementary Material). It was also impossible to achieve a diagnosis based on the IgM activity alone as ISR values were often very close in value and could not be directly compared between viruses due to test composition and result parameters. Analysis of the PRNT data showed no correlation between IgM activity with neutralizing activity. There was also no correlation between IgM positivity and PRNT positivity at any serum dilution. As most of the epidemiological data being generated from lower- and middle-income countries is based on IgM diagnostics, without further confirmatory testing or collection of convalescent serum, this raises questions as to the true prevalence for these viruses.

Similar diagnostic issues were present for neutralizing antibodies as most patients exhibited some level of neutralization for WNV, JEV, and DENV 1-4. Similar neutralizing activity has been reported for DENV and Zika virus, WNV and Murray Valley encephalitis virus, JEV with DENV 1-4, and Yellow fever virus with WNV, DENV, and tick-borne encephalitis virus (25-28). We also noted a large background of prior DENV exposure indicated via highly neutralizing PRNT titers, which mirrors what has been reported in other studies examining flaviviral cross-reactivity $(26,28)$.

When following the CDC/WHO diagnostic algorithms for arboviral disease, cross-reactivity devalues their use if IgM ELISA and PRNT are used as diagnostic tools. As stated by Lee et al. "Prior to the introduction of Zika to the Americas, PRNT was considered the gold standard of serological assays" (26). Cross-reactive samples used to be diagnosed as "flavivirus exposed" or some other generic classification. Since effective treatments are not available for most flavivirus infections, a definitive diagnosis was not viewed as significant as it is growing to be. Emerging data documenting debilitating sequelae including arthralgia and neurological issues, the development of neurodegenerative disorders, and horrific congenital consequences emphasize the need for rapid and accurate diagnostics to drive decisions and reassure patients and health care providers $(26,29-33)$.

Dengue viruses are endemic in Pakistan, and as such, many patients enrolled in this study were diagnosed as having DENV fever by physicians although neurological symptoms were present. It is not uncommon for DENV to co-circulate WNV and/ or JEV, which can lead to misdiagnosis as most of the symptoms overlap. These viruses present as a flu-like illness characterized by fever, myalgia, headache, gastrointestinal disturbance, and maculopapular rash. Up to $1 \%$ of $\mathrm{WNV}$-/JEV-infected individuals may show neuroinvasive disease $(34,35)$. Clinical features like headache, altered mental status, GI distress, limb weakness, and motor neuron disease pattern with flaccid paralysis are clues to the diagnosis of CNS infection with WNV or JEV (34, 35). Over a third of WNV-confirmed patients here had clinical features consistent with neuroinvasive disease. WNV-exposed patients exhibited unique symptoms when compared to JEV. WNV-infected individuals were much more likely to exhibit altered mental status, reduced Glasgow Coma scores, encephalitis, and seizure activity, whereas persons with JEV infection were more likely to suffer from nausea, thrombocytopenia, and be male.

The data show that average summer temperatures correlated with human WNV incidence. Mosquito numbers and human $\mathrm{WNV}$ infections and precipitation were also directly correlated. It was during this time that WNV was detected in a pool of Culex spp., via RT-PCR, which suggests that active WNV transmission was occurring. These findings are concordant with similar reports in Europe $(35,36)$. The city of Karachi is an ideal urban environment for Culex spp. propagation and transmission. The city has vectors, hosts, and an abundance of standing water with sewage or other organic material that enhance larval propagation (37-39). The low numbers of Aedes and Anopheles spp. collected was most likely due to our trapping methods. No trapping occurred during the daytime when Aedes mosquitoes are active and $\mathrm{CO}_{2}$ or other baits for attracting Anopheles were not used (40). Further surveillance of larger geographical areas along with larval and pupal demographic surveys are required to assess overall picture of the various mosquito species in Karachi.

A major limitation of this study was that only patient serum was tested via RT-PCR for the presence of virus. Since the inception of this study, research in molecular diagnostics of clinical specimens has determined that whole blood and urine are more useful for detecting WNV in human patients (41). Another limitation of the study is that vector surveillance was conducted in a small geographic region of Karachi $\left(0.34 \mathrm{~km}^{2}\right)$, and sentinel animals were not used. Despite these limitations, our results can be generalized as most of Karachi has similar features in terms of mosquito propagation sites, avian fauna, and susceptible human population.

Detection of vectors and humans infected with WNV is suggestive of a competent enzootic cycle. The climatic and 
sanitation conditions in Karachi are supportive of active propagation and dissemination of vectors and consequent risk of an epidemic outbreak of this virus. Active disease surveillance and preventive strategies are not available in Pakistan but are urgently needed at regional and national levels to prevent such from happening in timely manner. Moreover, there is as a great need for the standardization of diagnostic assays and procedures, more accurate diagnostic assays, and renovated diagnostic algorithms for Pakistan and other countries where WNV co-circulates with other flaviviruses. While development of surveillance networks, diagnostic assays, and standardized procedures can be overwhelming for developing countries, it is rapidly becoming apparent that the long-term costs to public health are greater in the face of flavivirus disease. Given the high seroprevalence of flaviviruses, reinstitution of DEWS or similar program should be seriously considered for Pakistan.

Regardless, the findings of this study are an alert for physicians to suspect WNV infection in patients with altered mental status and other neurological symptoms in a dengue-like illness in patients inhabiting or traveling to and from Pakistan, especially during the late summer and autumn months. WNV-associated neuroinvasive disease is an important differential diagnosis for other neurological diseases such as poliovirus currently circulating in Pakistan.

\section{ETHICS STATEMENT}

Patients were enrolled under informed consent procedures that were reviewed and approved by the Ethics Review Committee, Aga Khan University (3183-PAT-ERC-14) and the Institutional Review Board, University of Florida (201500908). All enrolled subjects gave written informed consent in accordance with the Declaration of Helsinki.

\section{REFERENCES}

1. Londono-Renteria B, Colpitts TM. A brief review of West Nile virus biology. Methods Mol Biol (2016) 1435:1-13. doi:10.1007/978-1-4939-3670-0_1

2. Gubler DJ. Human arbovirus infections worldwide. Ann N Y Acad Sci (2001) 951:13-24. doi:10.1111/j.1749-6632.2001.tb02681.x

3. Rizzoli A, Jimenez-Clavero MA, Barzon L, Cordioli P, Figuerola J, Koraka $\mathrm{P}$, et al. The challenge of West Nile virus in Europe: knowledge gaps and research priorities. Euro Surveill (2015) 20:1-15. doi:10.2807/1560-7917. ES2015.20.20.21135

4. Roehrig JT. West Nile virus in the United States - a historical perspective. Viruses (2013) 5(12):3088-108. doi:10.3390/v5123088

5. Chancey C, Grinev A, Volkova E, Rios M. The global ecology and epidemiology of West Nile virus. Biomed Res Int (2015) 2015:376230. doi:10.1155/2015/376230

6. Akhter R, Hayes CG, Baqar S, Reisen WK. West Nile virus in Pakistan. III. Comparative vector capability of Culex tritaeniorhynchus and eight other species of mosquitoes. Trans R Soc Trop Med Hyg (1982) 76(4):449-53. doi:10.1016/0035-9203(82)90132-8

7. Hayes CG, Baqar S, Ahmed T, Chowdhry MA, Reisen WK. West Nile virus in Pakistan. 1. Sero-epidemiological studies in Punjab Province. Trans R Soc Trop Med Hyg (1982) 76(4):431-6. doi:10.1016/0035-9203(82)90130-4

8. Khan E, Farooqi JQ, Barr KL, Prakoso D, Nasir A, Kanji A, et al. Flaviviruses as a cause of undifferentiated fever in Sindh Province, Pakistan: a preliminary report. Front Public Health (2016) 4:8. doi:10.3389/fpubh.2016.00008

\section{AUTHOR CONTRIBUTIONS}

The following authors contributed to this manuscript in the following ways: contribution to the conception and design of the work: MTL, EK, KLB, JAL; acquisition, analysis and interpretation of data: MTL, EK, DP, KLB, KI, SA, ZYK, ZA, AA, JF, FM; drafting, editing, revising and approving drafts: MTL, KLB, EK, KI, DP, JF, FM, JAL. MTL, EK, and KLB all agree to be accountable for all aspects of the work.

\section{ACKNOWLEDGMENTS}

Special thanks to Naeem Akhtar Assistant Meteorologist Pakistan Meteorological Department, University Road Karachi for sharing the metrological data. We are grateful to Ms. Sally Beachboard, who has spent many hours determining supply routes for private vendors and in negotiating costs of supplies for our work in both the United States and Pakistan. We are grateful for the taxonomic keys provided by the Navy Entomology Center of Excellence, Jacksonville, Florida. We thank WRCEVA for sharing isolates of DENV and JEV.

\section{FUNDING}

This work was supported by the Defense Threat Reduction Agency, Basic Research Award HDTRA1-14-1-0022, to the University of Florida. The contents do not necessarily reflect the position or the policy of the federal government, and no official endorsement should be inferred.

\section{SUPPLEMENTARY MATERIAL}

The Supplementary Material for this article can be found online at https://www.frontiersin.org/articles/10.3389/fpubh.2018.00020/ full\#supplementary-material.

9. Zohaib A, Saqib M, Beck C, Hussain MH, Lowenski S, Lecollinet S, et al. High prevalence of West Nile virus in equines from the two provinces of Pakistan. Epidemiol Infect (2015) 143(9):1931-5. doi:10.1017/s0950268814002878

10. Balakrishnan A, Thekkekare RJ, Sapkal G, Tandale BV. Seroprevalence of Japanese encephalitis virus \& West Nile virus in Alappuzha district, Kerala. Indian J Med Res (2017) 146(Suppl):S70-5. doi:10.4103/ijmr.IJMR_1638_15

11. Elyan DS, Moustafa L, Noormal B, Jacobs JS, Aziz MA, Hassan KS, et al. Serological evidence of flaviviruses infection among acute febrile illness patients in Afghanistan. J Infect Dev Ctries (2014) 8(9):1176-80. doi:10.3855/ jidc. 4183

12. Aghaie A, Aaskov J, Chinikar S, Niedrig M, Banazadeh S, Mohammadpour HK. Frequency of West Nile virus infection in Iranian Blood Donors. Indian J Hematol Blood Transfus (2016) 32(3):343-6. doi:10.1007/s12288-015-0567-5

13. Chinikar S, Javadi A, Ataei B, Shakeri H, Moradi M, Mostafavi E, et al. Detection of West Nile virus genome and specific antibodies in Iranian encephalitis patients. Epidemiol Infect (2012) 140(8):1525-9. doi:10.1017/ s0950268811002056

14. Khan SA, Chowdhury P, Choudhury P, Dutta P. Detection of West Nile virus in six mosquito species in synchrony with seroconversion among sentinel chickens in India. Parasit Vectors (2017) 10(1):13. doi:10.1186/s13071-016-1948-9

15. Kalaiyarasu S, Mishra N, Khetan RK, Singh VP. Serological evidence of widespread West Nile virus and Japanese encephalitis virus infection in native domestic ducks (Anas platyrhynchos var domesticus) in Kuttanad region, Kerala, India. Comp Immunol Microbiol Infect Dis (2016) 48:61-8. doi:10.1016/j.cimid.2016.08.002 
16. Mishra N, Kalaiyarasu S, Nagarajan S, Rao MV, George A, Sridevi R, et al. Serological evidence of West Nile virus infection in wild migratory and resident water birds in Eastern and Northern India. Comp Immunol Microbiol Infect Dis (2012) 35(6):591-8. doi:10.1016/j.cimid.2012.08.002

17. Fereidouni SR, Ziegler U, Linke S, Niedrig M, Modirrousta H, Hoffmann B, et al. West Nile virus monitoring in migrating and resident water birds in Iran: are common coots the main reservoirs of the virus in wetlands? Vector Borne Zoonotic Dis (2011) 11(10):1377-81. doi:10.1089/vbz.2010.0244

18. Ahmadnejad F, Otarod V, Fallah MH, Lowenski S, Sedighi-Moghaddam R, Zavareh A, et al. Spread of West Nile virus in Iran: a cross-sectional serosurvey in equines, 2008-2009. Epidemiol Infect (2011) 139(10):1587-93. doi:10.1017/ s0950268811000173

19. Chinikar S, Shah-Hosseini N, Mostafavi E, Moradi M, Khakifirouz S, Jalali T, et al. Seroprevalence of West Nile virus in Iran. Vector Borne Zoonotic Dis (2013) 13(8):586-9. doi:10.1089/vbz.2012.1207

20. Abbas T, Xu Z, Younus M, Qayyum A, Riaz MT. Seasonality in hospital admissions of Crimean-Congo hemorrhagic fever and its dependence on ambient temperature-empirical evidence from Pakistan. Int J Biometeorol (2017) 61(11):1893-7. doi:10.1007/s00484-017-1359-4

21. Yaqub T, Shabbir MZ, Mukhtar N, Tahir Z, Abbas T, Amir E, et al. Detection of selected arboviral infections in patients with history of persistent fever in Pakistan. Acta Trop (2017) 176:34-8. doi:10.1016/j.actatropica.2017. 07.019

22. Naqvi S, Bashir S, Rupareliya C, Shams A, Giyanwani PR, Ali Z, et al. Clinical spectrum of chikungunya in Pakistan. Cureus (2017) 9(7):e1430. doi:10.7759/ cureus. 1430

23. Centers. for. Disease. Control and prevention. Arboviral Diseases, Neuroinvasive and Non-neuroinvasive 2015 Case Definition. Atlanta: Department of Health \& Human Services (2017).

24. Barros SC, Ramos F, Zé-Zé L, Alves MJ, Fagulha T, Duarte M, et al. Simultaneous detection of West Nile and Japanese encephalitis virus RNA by duplex TaqMan RT-PCR. J Virol Methods (2013) 193(2):554-7. doi:10.1016/j. jviromet.2013.07.025

25. Piyasena TBH, Setoh YX, Hobson-Peters J, Prow NA, Bielefeldt-Ohmann H, Khromykh AA, et al. Differential diagnosis of flavivirus infections in horses using viral envelope protein domain III antigens in enzyme-linked immunosorbent assay. Vector Borne Zoonotic Dis (2017) 17(12):825-35. doi:10.1089/ vbz.2017.2172

26. Lee WT, Wong SJ, Kulas KE, Dupuis AP II, Payne AF, Kramer LD, et al. Development of zika virus serologic testing strategies in New York state. J Clin Microbiol (2017):01591-17. doi:10.1128/jcm.01591-17

27. Li J, Gao N, Fan D, Chen H, Sheng Z, Fu S, et al. Cross-protection induced by Japanese encephalitis vaccines against different genotypes of dengue viruses in mice. Sci Rep (2016) 6:19953. doi:10.1038/srep19953

28. Mansfield KL, Horton DL, Johnson N, Li L, Barrett ADT, Smith DJ, et al. Flavivirus-induced antibody cross-reactivity. JGen Virol (2011) 92(Pt 12):2821-9. doi:10.1099/vir.0.031641-0

29. Barber LM, Schleier JJ, Peterson RK. Economic cost analysis of West Nile virus outbreak, Sacramento County, California, USA, 2005. Emerg Infect Dis (2010) 16(3):480-6. doi:10.3201/eid1603.090667
30. Yeung MW, Tomlinson G, Loeb M, Sander B. Health-related quality of life in persons with West Nile virus infection: a longitudinal cohort study. Health Qual Life Outcomes (2017) 15:210. doi:10.1186/s12955-017-0787-5

31. Ronca SE, Dineley KT, Paessler S. Neurological sequelae resulting from Encephalitic alphavirus infection. Front Microbiol (2016) 7:959. doi:10.3389/ fmicb.2016.00959

32. Ronca SE. A cross sectional study of neurocognaitive outcomes Post-West Nile virus infection. Annual Meeting of the American Society of Tropical Medicine and Hygiene. Baltimore, MD: ASTMH (2017).

33. Earnest MP, Goolishian HA, Calverley JR, Hayes RO, Hill HR. Neurologic, intellectual, and psychologic sequelae following western encephalitis. A follow-up study of 35 cases. Neurology (1971) 21(9):969-74. doi:10.1212/ WNL.21.9.969

34. Debiasi RL, Tyler KL. West Nile virus meningoencephalitis. Nat Clin Pract Neurol (2006) 2(5):264-75. doi:10.1038/ncpneuro0176

35. Marcantonio $\mathrm{M}$, Rizzoli $\mathrm{A}$, Metz $\mathrm{M}$, Rosà $\mathrm{R}$, Marini $\mathrm{G}$, Chadwick $\mathrm{E}$, et al. Identifying the environmental conditions favouring West Nile virus outbreaks in Europe. PLoS One (2015) 10(3):e0121158. doi:10.1371/journal. pone.0121158

36. Deichmeister JM, Telang A. Abundance of West Nile virus mosquito vectors in relation to climate and landscape variables. J Vector Ecol (2011) 36(1):75-85. doi:10.1111/j.1948-7134.2011.00143.x

37. Day JF. Mosquito oviposition behavior and vector control. Insects (2016) 7(4):1-22. doi:10.3390/insects7040065

38. McVey DS, Wilson WC, Gay CG. West Nile virus. Rev Sci Tech (2015) 34(2):431-9. doi:10.20506/rst.34.2.2369

39. Feroz D, Hadi R. Species diversity and abundance of resident and migratory bird fauna of a North-Western Peri-Urban Area. Karachi Int J Fauna Biol Studies (2016) 3(3):175-180.

40. Lima JB, Rosa-Freitas MG, Rodovalho CM, Santos F, Lourenço-de-Oliveira R. Is there an efficient trap or collection method for sampling Anopheles darlingi and other malaria vectors that can describe the essential parameters affecting transmission dynamics as effectively as human landing catches? - A review. Mem Inst Oswaldo Cruz (2014) 109(5):685-705. doi:10.1590/0074-0276140134

41. Nagy A, Ban E, Nagy O, Ferenczi E, Farkas A, Banyai K, et al. Detection and sequencing of West Nile virus RNA from human urine and serum samples during the 2014 seasonal period. Arch Virol (2016) 161(7):1797-806. doi:10.1007/s00705-016-2844-5

Conflict of Interest Statement: The authors declare that the research was conducted in the absence of any commercial or financial relationships that could be construed as a potential conflict of interest.

Copyright (C) 2018 Khan, Barr, Farooqi, Prakoso, Abbas, Khan, Ashi, Imtiaz, Aziz, Malik, Lednicky and Long. This is an open-access article distributed under the terms of the Creative Commons Attribution License (CC BY). The use, distribution or reproduction in other forums is permitted, provided the original author(s) and the copyright owner are credited and that the original publication in this journal is cited, in accordance with accepted academic practice. No use, distribution or reproduction is permitted which does not comply with these terms 